disorders of speech and language (DD-L), and developmental disorders of scholastic skills (DDSch).

To describe care organization for these disorders in 7 Western countries.

Methodology: EBM literature review including systematic reviews, meta-analyses, randomized controlled trials and quasi-experimental studies.

Questionnaires sent to renowned experts in France, Germany, the Netherlands, Finland, UK, and Switzerland; description of the Belgian situation.

Results: In the short term, speech and language interventions for expressive phonological or vocabulary difficulties are effective; long term effects are not well-studied. In a few studies, no difference was found between treatment by trained parents or clinicians, or between individual and group therapy. For treatment of receptive language difficulties, only few studies are available suggesting little effects. Effective reading programs for reading disabilities should include training of the alphabetic principle and phonological awareness, and integration of these elements with comprehension and fluency skills. For mathematical disorders, too few publications are available to conclude.

Very few studies evaluated treatment intensity and duration, or the differences between monoand multidisciplinary treatment. Publications on treatment of co-morbid developmental or behavioral disorders were also lacking.

In most countries, multidisciplinary treatment (2 disciplines or more) is almost standard for DD-L and DD-Sch. Governmental responsibility for organization and funding belongs mostly to health and educational departments together.

Conclusion: It seems standard in Western societies to provide multidisciplinary treatment for DD-L and DD-Sch, but effectiveness of this strategy has been little evaluated.
1431

\section{PRETERM BIRTH AND SCHOOL PERFORMANCE: THE ROLE OF PARENTAL EDUCATION}

M. Gisselmann ${ }^{1}$, I. Koupil ${ }^{1}$, B.L. De Stavola ${ }^{2}$

${ }^{1}$ Centre of Health Equity Studies, CHESS, Stockholm University/Karolinska Institutet, Stockholm, Sweden, ${ }^{2}$ MSU (Medical Statistics Unit), Department of Epidemiology and Population Health, London School of Hygiene and Tropical Medicine, University of London, London, UK

While social background and birth characteristics are often found to be independently associated with school achievements the underlying mechanisms are still unclear. This study aims to explore the combined influence of parental education and gestational age grades in Mathematics in a cohort of Swedish children in whom we have previously shown associations of preterm birth with language skills. We studied 10825 children born 1973-1981, the third generation of the register-based Uppsala Multigenerational Birth Cohort. Logistic regression models were fitted to estimate odds ratios of achieving higher grades in Mathematics at age 16, relative to lower grade, by parental education and own gestational age. Our results shows that for children from families with lower parental education, the adjusted OR of receiving a higher grade was significantly lower for preterm compared to full term births grade in Mathematics (OR=0.53, 95\% Cl 0300.93). Estimate did not change substantially when adjusted for several potential confounders. There was no evidence of significant effects of shorter gestational age for children with parents from other educational groups. Consistent with our earlier results concerning language skills, we concluded that the disadvantage of shorter gestational age on the chance of achieving higher grade in Mathematics was confined to children from families where none of the parents had higher education. This suggests that the generally detrimental influence of shorter gestational age on school performance may be avoidable. 